\title{
Cutaneous Emulsion Dosage Form
}

National Cancer Institute

\section{Source}

National Cancer Institute. Cutaneous Emulsion Dosage Form. NCI Thesaurus. Code C149413.

Liquid, usually multidose preparation consisting of an emulsion intended for cutaneous use. 